J. Lake Sci.(湖泊科学), 2009, 21(2): 264-271

http://www.jlakes.org. E-mail: jlakes@niglas.ac.cn

(C2009 by Journal of Lake Sciences

\title{
多时相遥感影像提取湖泊边界信息的融合算法*
}

\author{
蒋锦刚 1,2 , 李爱农 2,4 , 邓 伟 2 , 宋孟强 2 , 冯文兰 1 , 李晓铃 2,3 \\ (1: 成都信息工程学院环境工程系, 成都 610225) \\ (2: 中国科学院、水利部成都山地灾害与环境研究所, 成都 610041) \\ (3: 西南交通大学土木工程学院, 成都 610031) \\ (4: Deptment of Geography, University of Maryland, College Park, MD 20742, USA)
}

摘 要: 为了有效改善遥感影像提取湖泊边界信息的可靠性和精度, 减少人为误差, 提出了一种利用多时相遥感影像提取边 界信息的加权平均融合算法以及误差的域法修正处理方法. 结果表明, 该方法能有效融合各时相影像信息，提高湖泊边界信 息提取的可靠性, 并且对融合时相变化较大的湖泊边界都有一定的普适性. 通过融合算法提取的呼伦湖面积为 $1928.35 \mathrm{~km}^{2}$, 修正后的面积为 $1929.85 \mathrm{~km}^{2}$. 通过利用地统计学理论对算法的验证及误差分析，得出相对误差空间变异拟合模型的块金方差 与基台值之比都小于 $25 \%$, 具有很强的空间相关性, 修正后的数据空间相关性要优于融合数据, 空间变程也得到了有效地降低, 显示修正后的数据对半方差函数理论模型的拟合程度更好

关键词: 湖泊边界; 多时相遥感影像; 数据融合; 误差修正; 空间变异

\section{Fusion algorithm for the information of lake boundary integration from multi-temporal remote sensing images}

\author{
JIANG Jingang ${ }^{1,2}$, LI Ainong ${ }^{2,4}$, DENG Wei ${ }^{2}$, SONG Mengqiang ${ }^{2}$, FENG Wenlan ${ }^{1} \&$ LI Xiaoling ${ }^{2,3}$ \\ (1: Department of Environmental Engineering, CUIT, Chengdu 610225, P.R. China) \\ (2: Institute of Mountain Hazards and Environment, CAS, Chengdu 610041, P.R. China) \\ (3: School of Civil Eng, Southwest Jiaotong University, Chengdu 610031, P.R. China) \\ (4: Department of Geography, University of Maryland, College Park, MD 20742, USA)
}

Abstract: For improving the reliability and accuracy of lake boundary integration information from remote sensing images and reducing human error, this paper put forwards a weighted average algorithm for integrating of the border information extracted by using the multi-temporal remote sensing images, and a processing approach of error interzone correction which can integrate the various temporal information effectively and improve the reliability of extracting the lake border information. According to this fusion algorithm the area of Lake Hulun is $1928.35 \mathrm{~km}^{2}$. Using the geostatistics theory to validate the errors, the area is $1929.85 \mathrm{~km}^{2}$. The results illuminated that the ratio of nugget to sill is less than $25 \%$, and the spatial correlation of the corrected data is superior to that of the integrated data. Furthermore the spatial variation range has been reduced effectively, and the extent of fitting the theoretical model is better in the corrected data than the original data.

Keywords: Lake border; multi-temporal remote sensing images; data integration; error correction; spatial variability; Lake Hulun

对湖泊面积和边界进行及时和精确的测定，为人类了解湖泊资源现状，揭示自然因素及人类活动对

* 中国科学院“西部之光”重点项目“若尔盖高原湿地动态变化遥感监测体系研究” $\left(08 \mathrm{R}_{2} 130130\right)$ 、国家自然科学基金“973”项目“黄 淮海地区湿地水生态过程、水环境效应及生态安全调控”(2006CB403301)和“中国湖泊水质、水量和生物资源调查”专项 (2006FY110600)子项目: 云贵高原湖泊卫星遥感调查 ( 08K0010010) 联合资助. 2008-09-13 收稿; 2008-12-07 收修改稿. 蒋 锦刚, 男, 1981 年生, 硕士研究生; E-mail: gangzg@yahoo.com.cn. 
湖泊水域的影响规律，以及合理开发利用、保护和治理湖泊水域资源有着极其重要的意义. 从1958年到 1987年, 我国陆续开展了全国第一次湖泊资源调查，获得大量湖泊基础数据，取得了良好的社会和经济 效益. 由于当时调查技术手段的限制, 第一次湖泊资源调查在湖泊位置和数量的调查上大都以人工实地 调查为主. 人工调查虽然能在第一线获取湖泊地面资料, 但是在确定湖泊边界、湖泊位置的精度上存在 不足; 而且我国幅员辽阔，有很多地方自然条件严酷，人工一线作业困难，致使第一次湖泊调查缺失了 许多湖泊信息. 近年来, 随着经济建设发展, 人为对自然湖泊的扰动增加, 加之全球气候变化的显著影 响, 我国湖泊萎缩严重, 湖泊边界变化明显, 有些湖泊已经或正在消失. 2007年, 国家科技基础性工作专 项重点项目“中国湖泊水质、水量和生物资源调查”正式启动，标志着对我国湖泊资源的第二次调查进人 了系统实施的新阶段. 此次调查在第一次湖泊调查工作基础上，将针对我国不同区域湖泊资源与环境变 化的特点, 以掌握我国湖泊水量、水质与生物资源变化及其形成原因为核心，以野外调查、定位观测和 遥感监测为手段, 以全国湖泊遥感普查与外流湖区主要湖泊重点调查为主要途径, 对全国面积大于 $1.0 \mathrm{~km}^{2}$ 的湖泊, 进行湖泊数量、面积和分布现状进行遥感调查, 为全面、系统地掌握我国湖泊现状及其变 化情况, 为国家经济可持续发展和生态安全, 提供可靠的、系统的、规范的科学数据和决策依据.

利用遥感(Remote Sensing, RS)对地观测技术进行湖泊调查, 具有覆盖面广、宏观性强、快速、多时相、 丰富的综合信息等优点，湖泊遥感调查结合地面实地验证能够有效保证湖泊调查的空间精度和及时更新的 需要, 大大降低了调查成本. 目前, 利用卫星遥感图像提取湖泊边界和面积等信息要素已成为研究湖泊的 重要手段之一 ${ }^{[1-5]}$. 随着人们对遥感研究的不断深人, 利用遥感影像自身的光谱信息, 对水陆混合特征像元 进行识别和提取的算法研究已成为湖泊边界提取的热点 ${ }^{[6-8]}$. 然而目前已有的一些湖泊边界自动提取的算 法不能很好地解决混合像元问题, 并且有些算法只针对一些典型的湖泊, 没有普适性,不能推广到其他湖区. 而对多时相遥感数据提取湖泊边界时，如何有效利用各时相信息方面的研究就更少了. 然而，由于遥感成 像的复杂性以及受多种不可控制因素的影响，其获取的数据具有不确定性的特征; 利用地理信息系统 (Geographic Information System, GIS)提取湖泊边界数据时, 在对遥感数据进行解译处理时也会存在不确定 性. 例如, 单时相遥感数据提取湖泊边界容易受时相影响; 遥感图像的空间纠正存在可允许误差; 存在混合 像元，使得湖泊水边界提取存在不确定性，即使是人工判读同样受制于混合像元和水位变迁的影响，而且 不同判译人员的知识和经验的差异也会带来湖泊边界提取的不确定性. 这就使得有必要发展一种方法来有 效降低这种不确定性. 针对RS/GIS空间数据信息的不确定性, 目前研究的主要方法有空间统计学、模糊集 理论、粗集理论、遗传算法、反思模型、误差摘模型 $[9-12]$ 等. 综合国内外对 RS/GIS空间数据模糊不确定性的 处理研究现状, 结合实践发展减小空间数据模糊不确定性的算法仍然非常必要.

为了改善提取湖泊信息的可靠性和精度, 减少人为误差, 通常需要选择多景时相较近的影像进行综 合考虑，以提取这一时期的湖泊信息. 如何有效综合所选取影像的信息，减少人工判读在提取湖泊边界 时的不确定性因素, 有必要发展一种算法来融合多时相遥感数据提取的湖泊边界信息. 本文在利用 CBERS遥感影像提取湖泊边界时，提出了一种多时相遥感影像提取矢量信息的加权平均融合算法以及误 差的域法修正处理方法. 通过对算法的验证及误差分析，得出该算法能有效融合各时相数据信息，并且 能降低数据的空间变异程度.

\section{1 多时相边界数据的融合算法及验证}

利用 GIS 提取不同时相遥感影像的湖泊边界数据时, 是以线或者多边形的矢量数据结构来描述空间 实体的, 这种方式提取的数据结构的组织和编码如图 1 所示.

对提取的多组数据进行融合时, 首先要选择一个主时相数据, 主时相可随机选择, 通常可选择丰水 期为主时相，以主时相的每个坐标数据为中心，通过空间搜索，找出每个时相与主时相空间上欧氏距离 之和最小的两个点数据, 该两个点与主时相坐标会构成一个三角形, 以主时相坐标为顶点引三角形的一 条高线(即搜索出的两个点所在的边的高线), 高线与边的交点作为该时相参与融合的坐标数据. 融合后 的坐标数据是由中心数据加上计算出的各时相交点数据乘上对应的权值计算得到，如图 2 是该融合方法 提取坐标的几何示意图, 其中 $O$ 是主时相的某一中心点, $A, B, C, D$ 分别为两个时相与主时相空间上欧氏 
距离之和最小的两个点, $O N, O M$ 分别是 $\triangle O A B$, 和 $\triangle O C D$ 的 $A B$ 边和 $C D$ 边上的高, 那么点 $O 、 M 、 N$ 就 是需要参与融合的坐标数据. 利用该方法进行各时相数据的融合, 可以有效将融合所得线上的点沿湖岸 线的法线方向进行融合, 避免沿湖岸线切线方向融合而增加误差, 并且可以在一定程度上克服人机交互 过程中不同线上矢量点步长选择的随意性，但各时相在矢量时步长选择应该尽量均匀.

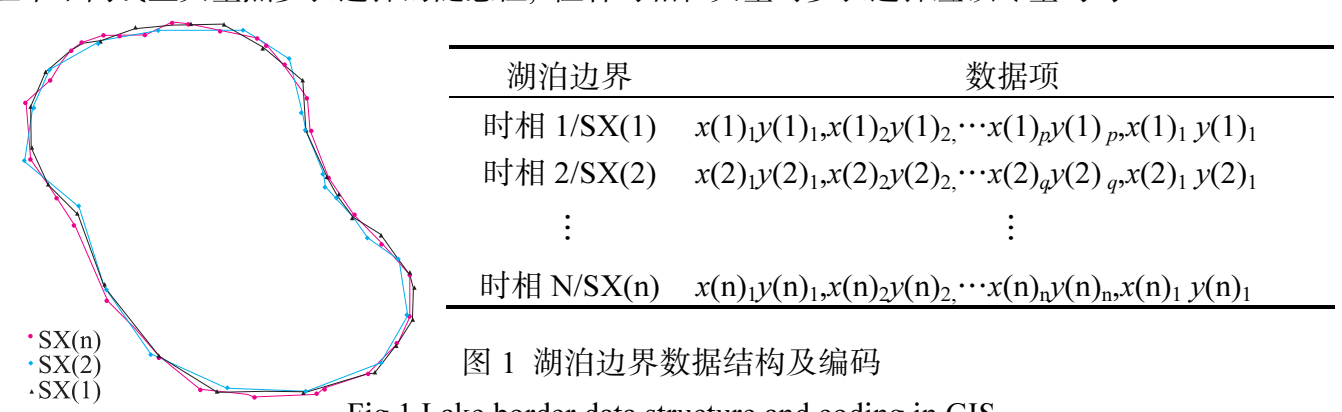

Fig.1 Lake border data structure and coding in GIS

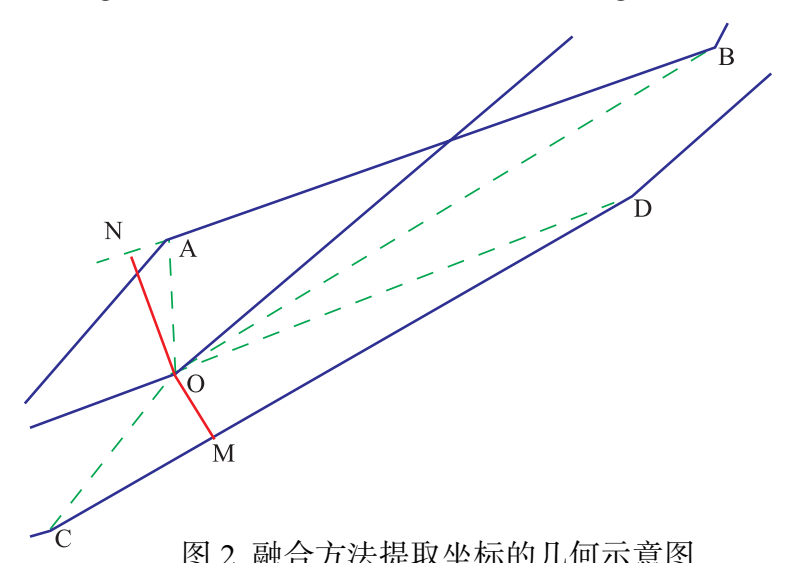

图 2 融合方法提取坐标的几何示意图

Fig.2 Geometric diagram of geographical coordinates for data integration model

这里选择时相 1 作为主时相，具体计算公式如下:

$$
\left\{\begin{array}{l}
x_{i}=\sum_{j=1}^{n} \omega_{j} x(j)_{i} \quad i=1,2, \cdots p . \\
y_{i}=\sum_{j=1}^{n} \omega_{j} y(j)_{i} \quad i=1,2, \cdots p . \\
S_{i}=\min \sum_{j=2}^{n}\left(\sqrt{\left[x_{1}(j)_{i}-x(1)_{i}\right]^{2}+\left[y_{1}(j)_{i}-y(1)_{i}\right]^{2}}+\sqrt{\left[x_{2}(j)_{i}-x(1)_{i}\right]^{2}+\left[y_{2}(j)_{i}-y(1)_{i}\right]^{2}}\right) \\
\sum_{j=1}^{n} \omega_{j}=1 \\
{\left[x_{2}(j)_{i}-x_{1}(j)_{i}\right]\left[x(j)_{i}-x(1)_{i}\right]+\left[y_{2}(j)_{i}-y_{1}(j)\right]\left[y(j)_{i}-y(1)_{i}\right]=0}
\end{array}\right.
$$

式中: $x_{i}, y_{i}$ 为融合后的坐标值; $i$ 为主时相某一坐标节点; $n$ 为选择时相的个数; $\omega_{j}$ 对应 $j$ 时相的权值; $x(j)_{i}$, $y(j)_{i}$ 为 $j$ 时相对应 $i$ 节点构成三角形的高线与边的交点坐标值; $p$ 为主时相提取的坐标数目; $x(1)_{i}, y(1)_{i}$ 为对 应主时相 $i$ 节点的坐标值; $S_{i}$ 为搜索的各时相两点坐标数据相对主时相数据的空间欧氏距离之和; $x_{1}(j)_{i}$, $y_{1}(j)_{i}, x_{2}(j)_{i}, y_{2}(j)_{i}$ 为对应 $i$ 节点搜索出来的 $j$ 时相的两个点的坐标值.

为了消除特异值对融合后的数据统计特征的影响, 本文在对融合后数据进行处理时采用了域法的识 别和修正, 具体做法为:

(1)计算融合后的数据与主时相数据之间的空间欧氏距离, 记为相对误差 $\Delta S$;

设某点融合后的坐标 $A\left(x_{i}, y_{i}\right)$ 与主时相坐标 $B\left(x(1)_{i}, y(1)_{i}\right)$ 所对应的真实点的空间坐标为 $C\left(x_{i}, y_{i}\right)$ 则有: 


$$
\begin{aligned}
\Delta S_{i} & =\sqrt{\left[x(1)_{i}-x_{i}\right]^{2}+\left[y(1)_{i}-y_{i}\right]^{2}} \\
\Delta S_{i} & =|\overrightarrow{A B}|=|\overrightarrow{C B}-\overrightarrow{C A}|
\end{aligned}
$$

其中 $|\overrightarrow{C A}|$ 是该点融合后数据的实际误差, 显然 $|\overrightarrow{C A}|$ 是与 $\triangle S_{i}$ 和 $|\overrightarrow{C B}|$ 的大小和夹角有关的量. $\Delta S_{i}$ 的大小从某 种程度上是可以反映实际误差的情况的, 尤其是 $\triangle S_{i}$ 的值偏大时, 所以通过相对误差 $\Delta S$ 的大小来对融合 后的特异值进行识别处理是十分有意义的.

(2)统计出数据 $\Delta S$ 的平均值 $\overline{\Delta S}$ 和标准差 $s$, 定义域法识别特异值的区间为 $[\overline{\Delta S}-3 s, \overline{\Delta S}+3 s]$, 即对 $\Delta S$ 在区间 $[\overline{\Delta S}-3 s, \overline{\Delta S}+3 s]$ 外的融合数据都认定为特异值, 对应的融合坐标数据就需要修正.

(3)在对 GIS 空间数据位置不确定性的研究中, 线位的不确定采用误差带来度量 ${ }^{[13-17]}$, 这些误差带模 型都是在假定数据误差服从正态分布的基础上建立的，因此本文在对特异值的坐标数据进行修正时，采 取在 $[\overline{\Delta S}-3 s, \overline{\Delta S}+3 s]$ 的误差区间内进行正态分布的随机修正. 具体计算公式如下:

$$
\left\{\begin{aligned}
x_{i} & =\sum_{j=1}^{n} \omega_{j} x(j)_{i} \pm \text { randn } \quad i=1,2 \cdots l<p \\
y_{i} & =\sum_{j=1}^{n} \omega_{j} y(j)_{i} \pm \text { randn } \quad i=1,2 \cdots l<p \\
\Delta S_{i} & =\sqrt{\left[x_{i}-x(1)_{i}\right]^{2}+\left[y_{i}-y(1)_{i}\right]^{2}} \in[\overline{\Delta S}-3 s, \overline{\Delta S}+3 s]
\end{aligned}\right.
$$

式中: $x_{i}, y_{i}$ 为特异值的坐标数据; $i$ 为某一特异值的坐标节点; $n$ 为选择时相的个数; $\omega_{j}$ 对应 $j$ 时相的权值; $r a n d n$ 为服从正态分布的随机数; $l$ 为特异值的个数; $x(1)_{i}, y(1)_{i}$ 为主时相对应 $i$ 节点的坐标值; $\Delta S_{i}$ 修正后的 数据与主时相数据之间的空间欧氏距离.

为了验证融合和修正后的数据质量情况，本文利用空间统计学理论来对融合和修正后的相对误差 进行分析与评价. 空间统计学是依靠有序的模型描述无序事件, 根据不确定性和有限信息分析、评价和 预测空间数据 ${ }^{[18-19]}$.

空间统计学中, 数据变量的空间变异性可以通过半方差函数来刻画, 其函数的计算可用下式表示:

$$
r(h)=\frac{1}{2 N(h)} \sum_{i=1}^{N(h)}\left[Z\left(x_{i}\right)-Z\left(x_{i}+h\right)\right]^{2}
$$

式中: $r(h)$ 为半方差函数; $h$ 为样点空间间隔距离, 称为步长; $N(h)$ 为间隔距离为 $h$ 的样点数; $Z\left(x_{i}\right)$ 和 $Z\left(x_{i}+h\right)$ 分别 是区域化变量 $Z\left(x_{i}\right)$ 在空间位置 $x_{i}$ 和 $x_{i}+h$ 的实测值. 若以 $h$ 为横坐标, 以 $r(h)$ 为纵坐标绘制的函数曲线图, 称为 半方差函数曲线图, 通过对 $r(h)$ 坐标值的拟合可以得出相应的理论模型和模型参数, 通过对模型参数的分析 可以得出空间变异的特点. 常用的半方差函数拟合的理论模型有球状模型、指数模型和高斯模型等.

变异函数理论模型有 4 个重要的参数, 即基台值、变程、块金值和分维数, 其中前 3 个参数可以直接从 拟合的变异函数中得到. 块金值是由实验误差和小于实际取样尺度引起的变异，表示随机部分的空间异 质性，块金值大表明较小尺度上的某种过程不可忽视，基台值通常表示系统内的总变异. 从结构性因素 的角度来看, 块金值与基台值的比值可以表明系统变量的相关性的程度. 如果比值 $<25 \%$, 说明系统具有 强烈的空间相关性; 如果比值在 $25 \%-75 \%$ 之间, 说明系统具有中等的空间相关性; 若 $>75 \%$ 说明系统相 关性很弱 ${ }^{[20]}$. 变程是指变异函数达到基台值所对应的距离, 它表明相对误差数据的空间自相关范围.

\section{2 实例分析}

\section{1 遥感数据的选取与处理}

为了使该算法具有普适性, 本文选取了湖泊边界随时相变化较为典型的呼伦湖作为研究湖泊, 如图 3、4 所示. 呼伦湖位于呼伦贝尔盟满洲里市南郊, 跨新巴尔虎左旗和新巴尔虎右旗, 水位在 $545.33 \mathrm{~m}$ 时, 长 $93.0 \mathrm{~km}$, 最大宽 $41.0 \mathrm{~km}$, 平均宽 $25.2 \mathrm{~km}$, 面积 $2339.0 \mathrm{~km}^{2}$. 湖区属温带半干旱气候, 年平均气温为 $-0.1^{\circ} \mathrm{C}$, 多年平均降水量为 $319 \mathrm{~mm}^{[21]}$. 该湖在 $A 、 B 、 C 3$ 个区域有着各自不同的特点, $A 、 B$ 区湖泊边界变化都比 较大, 其中 $A$ 区更为复杂, $C$ 区基本没有变化.

为了尽可能的减少湖泊面积和水边线随季节性因素变动对提取数据的影响, 本文选取了 3 个时相相近 
的CBERS遥感图像数据来提取呼伦湖的湖泊边界. 选取的 3 期数据的时间分别为 2005 年 10 月 20 日、2006年 8 月 31 日、2006年 9 月 26 日, 数据的最大时相跨度为 1 年，且在 8 月至 10 月间，这个时期的水位数据基本处于湖 泊的平均水位左右. 对选取的遥感图像数据进行进一步的处理主要有图像合成和几何精纠正, 遥感图像数 据采用4、3、2 波段彩色合成, 水陆像元区分相对明显, 有利于湖泊边界的矢量提取. 利用 $1: 50000$ 地形图数 据对影像进行几何精纠正, 每景影像选取 30 个以上分布均匀且清晰可辨的控制点, 保证图像几何精纠正的 精度控制在 0.5 个像元范围内, 选择ALBERS等积投影和北京 1954 坐标系为地图参考基准.

\section{2 算法的执行及结果}

在对湖泊边界进行矢量化提取时，在ArcGIS9.2中采用人机交互的方式，依据对水陆混合像元的判读 分别提取各期的湖泊边界, 在矢量化过程中, 矢量步长选择较为均匀, 其选择步长大约为 10-20个像元. 如图3是各时期的假彩色合成图像以及矢量化提取的湖泊边界.

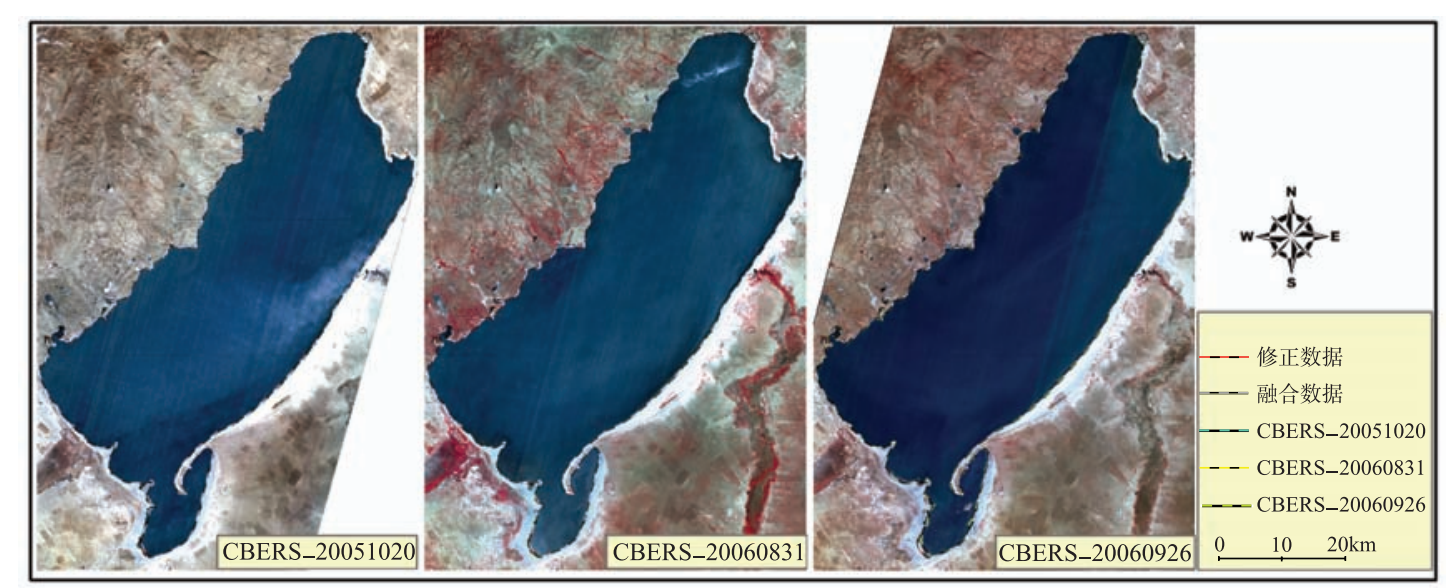

图3 假彩色合成(4、3、2波段)图像及矢量化处理的湖泊边界

Fig.3 The false color synthesize map of $4 、 3 、 2$ wave bands and vector processing of the border lake

在 ArcGIS9.2 环境中将各时相矢量存取的坐标数据导出, 在 MATLAB7.0 环境中编程实现对各期的湖 泊边界的融合, 由于该地区各月份的湖泊水位变化相对较大, 故对各时相数据进行赋权时, 可根据湖泊 边界的季节变化特点, 根据对应的影像的时相进行人为赋权, 这里 2006 年 8 月 31 日和 2006 年 9 月 26 日提取的湖泊数据的所附权值分别为 $0.4,2005$ 年 10 月 20 日的数据作为参考数据, 附权值较小为 0.2 , 然 后将融合和修正生成的数据在 ArcGIS9.2 环境中转成矢量的数据格式, 图 3、4 给出了融合和修正后的矢 量数据结果. 从图中可以看出, 融合和修正后的数据对各个时相的湖泊边界都能很好的吻合, 并且在提 取湖泊变化较大的区域其融合和修正的边界数据处于各时相之间.

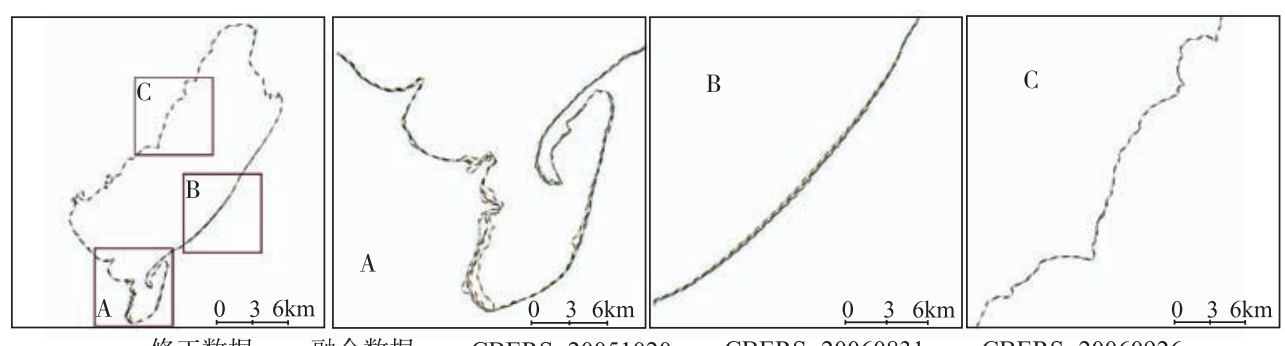

-.. 修正数据 - 融合数据 - - CBERS_20051020 - - CBERS_20060831 - CBERS_20060926

图4 融合和修正后的数据与各时相数据空间关系

Fig.4 The spatial relationships of each time data, integrated data and corrected data

表 1 是各时相提取的湖泊数据、融合数据和修正数据与《中国湖泊志》 ${ }^{[21]}$ 数据、2005-2006 年平均 
面积 ${ }^{[22-23]}$ 的比较, 由表可以看出融合数据和修正数据与 2005-2006 年面积平均的误差分别为 $1.13 \mathrm{~km}^{2}$ 和 $0.37 \mathrm{~km}^{2}$, 表明该算法在融合各时相数据时是十分有效的. 由于气候和人为因素的影响, 该湖的水域面积 和水位是在波动中逐渐减小的，其中 1999-2006 年湖泊面积就缩小了 $373.6 \mathrm{~km}^{2}$ 以上 ${ }^{[23]}$, 所以提取的湖泊 面积与湖泊志有较大的差异是客观存在的.

表 1 提取湖泊数据的相关参数比较

Tab.1 Comparing the relevant parameters of Hulun lakes

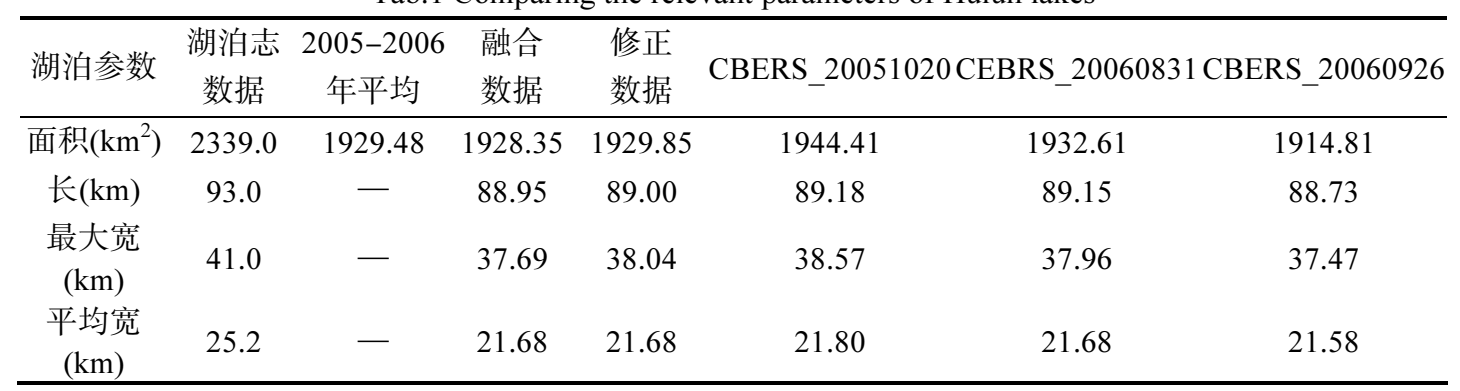

\section{3 融合误差的空间变异分析}

对融合后和修正后的相对误差分布选择 $A 、 B 、 C 3$ 个区域进行统计分析得出的结果如表 2 , 从表 2 可 以看出, $A$ 区域融合后和修正后的相对误差的变异系数分别为 $91.43 \%$ 和 $90.36 \%$, 属强变异; $B$ 区域融合后 和修正后的相对误差的变异系数分别为 $69.35 \%$ 和 $65.92 \%$, 属中等变异; $C$ 区域融合后和修正后的相对误 差的变异系数分别为 $55.34 \%$ 和 $52.22 \%$, 属中等变异. 域值修正处理后的平均值、中位数、标准方差、最 大值、变异系数都有不同程度的减小. 图 5 是对数据进行正态分布概率检验，得出经过域值修正处理后的误 差数据更符合正态分布.

表 2 融合后和修正后的相对误差的描述性统计

Tab.2 Descriptive statistics of integrated relative error and corrected relative error

\begin{tabular}{cccccccc}
\hline 区域 & 误差数据 & 平均值 & 中位数 & 标准方差 & 最小值 & 最大值 & 变异系数 $(\%)$ \\
\hline \multirow{2}{*}{$A$} & 融合数据 & 138.16 & 97.45 & 126.32 & 8.97 & 234.13 & 91.43 \\
& 修正数据 & 119.54 & 88.68 & 108.02 & 8.87 & 167.68 & 90.36 \\
$B$ & 融合数据 & 68.56 & 55.37 & 47.55 & 6.64 & 153.43 & 69.35 \\
& 修正数据 & 57.07 & 52.75 & 37.62 & 6.64 & 106.71 & 65.92 \\
& C融合数据 & 32.09 & 29.48 & 17.76 & 3.67 & 94.32 & 55.34 \\
& 修正数据 & 28.07 & 26.52 & 14.66 & 4.07 & 94.32 & 52.22 \\
\hline
\end{tabular}
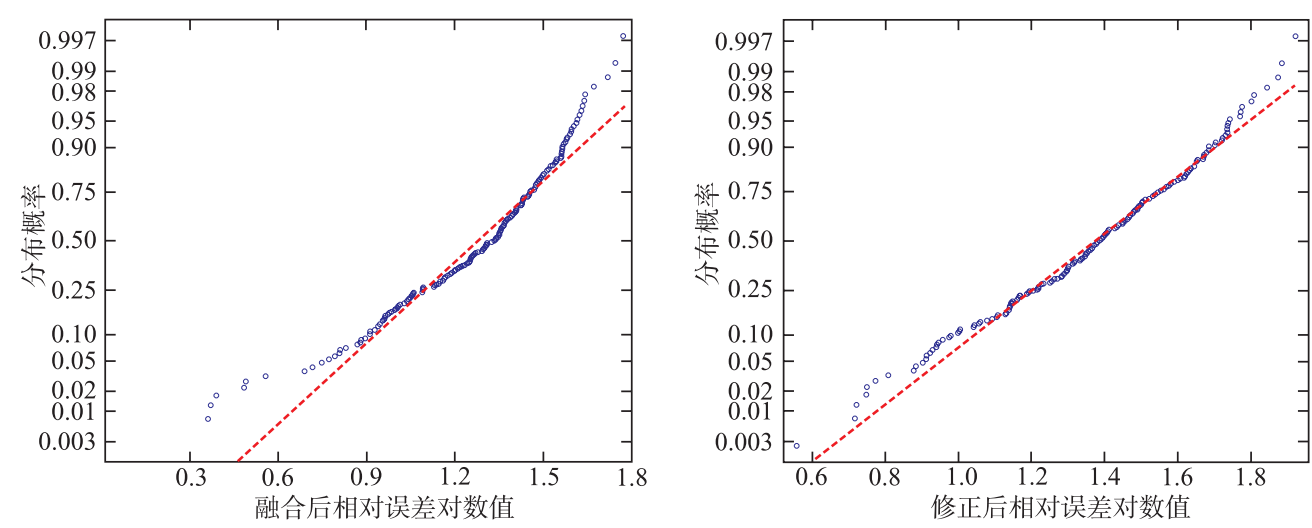

图 5 正态分布概率图

Fig.5 Normal probability distribution of integrated relative error and corrected relative error 
在对融合和修正相对误差进行空间变异分析时, 步长变化范围选择 $0-15 \mathrm{~km}$, 步长间距分别选择 $100 \mathrm{~m} 、 300 \mathrm{~m} 、 500 \mathrm{~m}$. 由图 6 和表 3 可以分析，拟合的各向同性的半方差函数的理论模型为高斯模型、指 数模型, 融合数据相对误差的半方差函数的理论模型块金方差 $C_{0}$ 在 0.085-0.100 之间变化, 基台值 $C_{0}+C$ 在 0.5710-0.5714 之间变化, 有效变程在 1.31-1.38km 之间变化, 块金方差与基台值之比 $C_{0} /\left(C_{0}+C\right)$ 在 $14.9 \%-17.5 \%$ 之间变化; 修正数据相对误差的半方差函数的理论模型块金方差 $C_{0}$ 在 $0.064-0.067$ 之间变 化, 基台值 $+C$ 在 0.4480-0.4510 之间变化, 有效变程在 0.87-0.92km 之间变化, 块金方差与基台值之比 $C_{0} /\left(C_{0}+C\right)$ 在 $14.2 \%-14.8 \%$ 之间变化.

分析表明, 融合和修正相对误差的块金方差与基台值之比都小于 $25 \%$, 两组数据都具有强的空间相 关性, 修正后的数据空间相关性要优于融合数据, 对误差数据进行域值修正后会有效降低数据的空间变 程, 并且修正后的数据对理论模型的拟合程度更好, 其决定系数都有明显的提高.
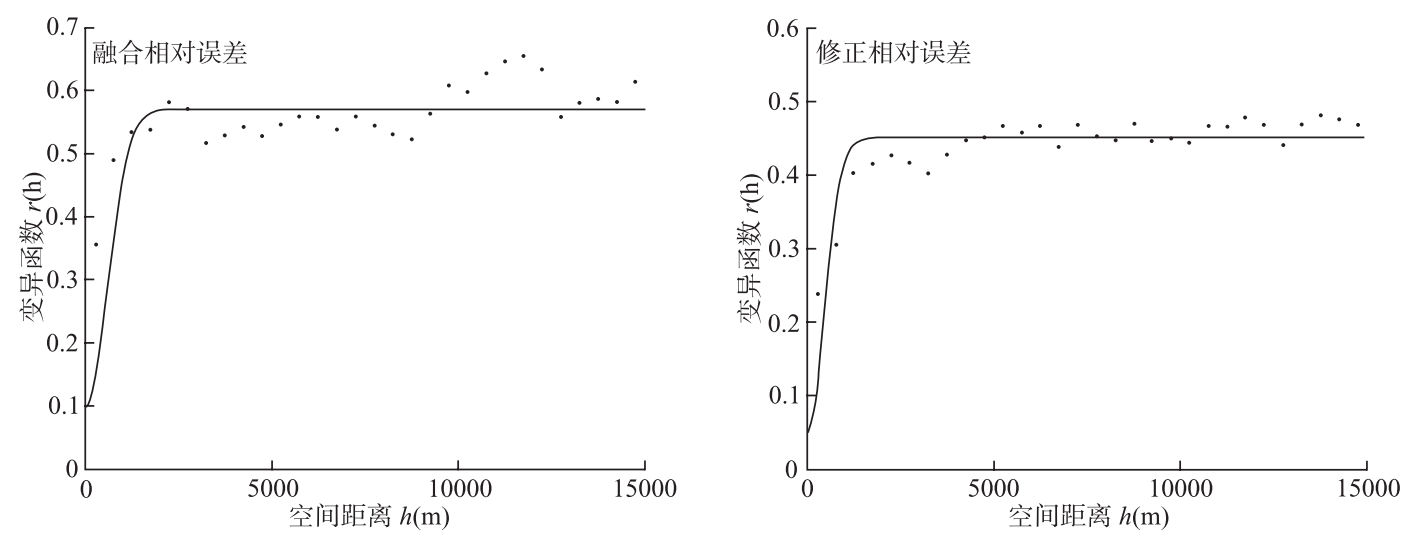

图 6 相对误差数据的变异函数曲线图(步长: $500 \mathrm{~m}$ )

Fig.6 Isotropic semivariograms of relative error data(Step: 500meters)

表 3 不同步长下的理论半方差函数极其参数

Tab.3 Theoretical semivariogram model parameters at various lag intervals

\begin{tabular}{|c|c|c|c|c|c|c|c|}
\hline $\begin{array}{c}\text { 相对误差 } \\
\text { 数据 } \\
\end{array}$ & $\begin{array}{c}\text { 步长距离 } \\
(\mathrm{m})\end{array}$ & 理论模型 & $C_{0}$ & $C_{0}+C$ & $\begin{array}{c}\text { 有效变程 } \\
(\mathrm{km})\end{array}$ & $C_{0} /\left(C_{0}+C\right)$ & 决定系数 $R^{2}$ \\
\hline \multirow[t]{3}{*}{ 融合数据 } & 100 & 高斯模型 & 0.085 & 0.5714 & 1.38 & 0.149 & 0.583 \\
\hline & 300 & 指数模型 & 0.100 & 0.5710 & 1.34 & 0.175 & 0.538 \\
\hline & 500 & 指数模型 & 0.100 & 0.5710 & 1.31 & 0.175 & 0.598 \\
\hline \multirow[t]{3}{*}{ 修正数据 } & 100 & 指数模型 & 0.064 & 0.4510 & 0.89 & 0.142 & 0.596 \\
\hline & 300 & 指数模型 & 0.067 & 0.4510 & 0.92 & 0.148 & 0.614 \\
\hline & 500 & 指数模型 & 0.065 & 0.4480 & 0.87 & 0.145 & 0.638 \\
\hline
\end{tabular}

\section{3 结论与讨论}

空间信息的不确定性是RS与GIS中一个普遍存在的问题, 利用多时相遥感数据信息来提取湖泊边界 特征信息, 可以弥补单一时相解译时诸多缺点, 提高遥感解译的可靠性和精度. 研究表明, 本文提出的 多时相遥感影像提取边界信息的加权平均融合算法以及误差的域法修正处理方法, 在综合利用多时相遥 感影像数据提取可靠的湖泊信息方面具有积极的意义，其算法具有以下优点:

(1)多时相遥感影像提取矢量信息的加权平均融合算法以及误差的域法修正处理方法能有效融合各 时相数据信息, 降低遥感解译过程中不确定性因素的影响, 各时相加权时的权值可根据实际情况人为赋 值, 赋权灵活. 利用融合后的数据与主时相数据之间的空间欧氏距离, 即相对误差, 对融合后的特异值 
进行识别处理意义明确; 采用域值区间的正态随机修正方法, 可以对识别出的特异数值进行有效修正.

(2)利用地统计学的知识对融合和修正后的数据进行空间变异分析，得出融合和修正的相对误差数据 都具有强的空间相关性, 数据之间的变异由结构性因素影响, 人为提取时的随机性因素较少. 修正后的 数据空间相关性要优于融合数据, 空间变程也得到了有效地降低, 并且修正后的数据对半方差函数理论 模型的拟合程度更好, 其决定系数有明显地提高.

同时研究还发现，在对特异值进行修正时采用正态分布随机修正，虽然能有效降低误差幅度，但可 能会出现修正后的矢量曲线在某些区间段出现锯齿形状. 此外在对各时相数据进行加权时, 需要根据水 位变化而定, 并且没有考虑各组数据之间的空间相关程度, 所附权值的大小受人为主观因素影响, 这都 需要在后续研究中进行完善.

\section{4 参考文献}

[1] 孟庆伟, 罗 鹏, 余 佳. 遥感技术在湖泊环境研究中的应用. 地质力学学报, 2006, 12(3): 287-293.

[2] 于雪英, 江 南. 基于RSGIS技术的湖面变化信息提取与分析一以艾比湖为例. 湖泊科学, 2003, 15(1): 81-84.

[3] 殷立琼, 江 南, 杨英宝. 基于遥感技术的太湖近15年面积动态变化. 湖泊科学, 2005, 17(2): 139-142.

[4] 施晶晶. 中巴资源一号卫星湖泊信息提取——南京景为例. 湖泊科学, 2001, 13(3): 280-284.

[5] 李俊杰, 何隆华, 戴锦芳等. 基于遥感影像纹理信息的湖泊围网养殖区提取. 湖泊科学, 2006, 18(4): 337-342.

[6] 万显荣, 舒 宁, 郑建生. 一种基于种子点与连通性分析的快速水体边界提取方法. 国土资源遥感, 2000, 46(4): 44-49.

[7] 胡争光, 王神婷, 池天河等. 基于混合像元分解和双边界提取的湖泊面积变化监测. 遥感信息, 2007, 3: 34-38.

[8] 粱益同, 胡江林. NOAA卫星图像水体信息神经网络识别方法的探讨. 应用气象学报, 2001, 12(1): 85-90.

[9] 胡圣武, 许 辉, 王新洲等. 遥感数据的模糊不确定性及其处理方法探讨. 地理与地理信息科学, 2004, 20(4): 19-22.

[10] 承继成, 郭华东, 史文中等. 遥感数据的不确定性问题. 北京: 科学出版社, 2004.

[11] 郭达志, 胡召玲, 陈云浩. GIS中空间对象的不确定性研究. 中国矿业大学学报, 2000, 29(1): 20-24.

[12] 李大军, 龚健雅, 谢刚生等. GIS中面元的误差熵模型. 测绘学报, 2003, 32(1): 31-35.

[13] Chirsman. A theory of cartographic error and its measurement in digital data bases. Auto-Carto, 1982: 5.

[14] Goodchild MF, Guoqing S, Shiren Y. Development and Test of an Error Model for Categorical data. International Journal of Geographical Information Systems, 1992, 6(2).

[15] 史文中. 空间数据误差处理的理论与方法. 北京: 科学出版社, 1998 .

[16] 刘文宝. GIS 空间数据的不确定性理论[博士学位论文]. 武汉: 武汉测绘科技大学, 1995.

[17] 游扬声. 一般分布模式下 GIS 位置数据的不确定性研究[博士学位论文]. 武汉: 武汉大学, 2005.

[18] 李德仁, 王树良, 李德毅等. 论空间数据挖掘和知识发现的理论与方法. 武汉大学学报(信息科学版), 2002, 27(3): 221-233.

[19] 张景雄, 杜道生, 孙家柄. 用随机方法建立矢量数据的误差模型. 武汉测绘科技大学学报, 2000, 25(1): 49-53.

[20] 王政权. 地统计学及在生态学中的应用. 北京: 科学出版社, 1999.

[21] 王苏明, 窦鸿身. 中国湖泊志. 北京: 科学出版社, 1998 .

[22] 赵慧颖, 乌力吉, 郝文俊. 气候变化对呼伦湖湿地及其周边地区生态环境演变的影响. 生态学报, 2008, 28(3): 1064-1071.

[23] 赵慧颖, 李成才, 赵恒和等. 呼伦湖湿地气候变化及其对水环境的影响. 冰川冻土, 2007, 29(5): 795-801. 